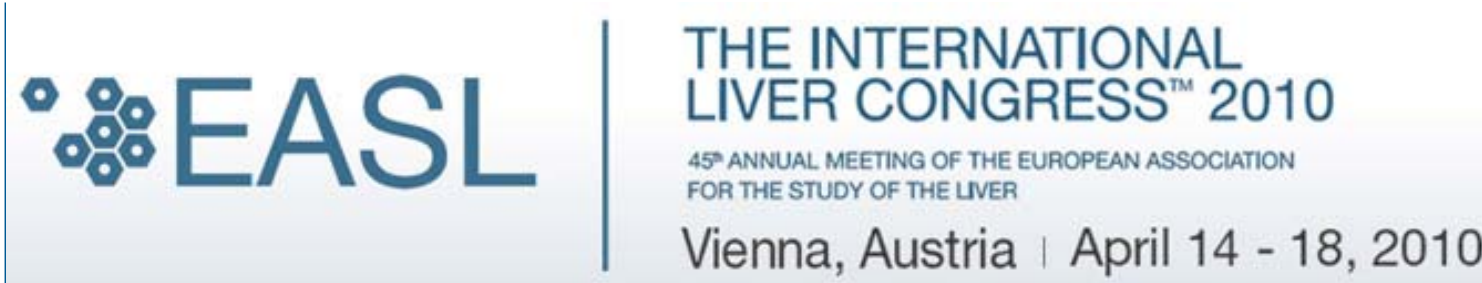

ORAL PRESENTATIONS

Session Title: Parallel Session: LATE-BREAKERS

Presentation Date: Apr 17, 2010

\title{
IFN STIMULATED GENE EXPRESSION IN THE LIVER IS A BETTER PREDICTOR OF TREATMENT RESPONSE IN CHRONIC HEPATITIS C THAN THE IL28B (IFN 33 ) GENOTYPE
}

M.T. Dill ${ }^{1}$, F.H.T. Duong ${ }^{1}$, J.E. Vogt ${ }^{1}$, S. Bibert ${ }^{2}$, P.-Y. Bochud ${ }^{2}$, L. Terracciano ${ }^{1}$, A. Papassotiropoulos ${ }^{1}$, V. Roth $^{1}$, M.H. Heim $^{1}$

${ }^{1}$ Department of Biomedicine, ${ }^{2}$ Division of Gastroenterology and Hepatology, University Hospital Basel, ${ }^{3}$ Computer Science Department, University of Basel, Basel, ${ }^{4}$ Infectious Diseases Service, Department of Medicine, Centre Hospitalier Universitaire Vaudois, ${ }^{5}$ Institute of Microbiology, University of Lausanne, Lausanne, ${ }^{6}$ Institute of Pathology, University Hospital Basel, ${ }^{7}$ Division of Molecular Psychology, Life Sciences Training Facility, Biozentrum, University of Basel, Basel, Switzerland.*michael.dill@unibas.ch

Background: Therapy of chronic hepatitis $\mathrm{C}(\mathrm{CHC})$ with pegIFNa/ribavirin achieves sustained virologic response (SVR) in $\sim 55 \%$. Pre-activation of the endogenous interferon system in the liver is associated non-response (NR). Recently, genome-wide association studies described associations of allelic variants near the IL28B (IFNA3) gene with treatment response and with spontaneous clearance of the virus. We investigated if the IL28B genotype determines the constitutive expression of IFN stimulated genes (ISGs) in the liver of patients with CHC.

Methods: We genotyped 93 patients with CHC for 3 IL28B single nucleotide polymorphisms (SNPs, rs 12979860 , rs8099917, rs12980275), extracted RNA from their liver biopsies and quantified the expression of IL28B and of 8 previously identified classifier genes which discriminate between SVR and NR (IFI44L, RSAD2, ISG15, IFI22, LAMP3, OAS3, LGALS3BP and HTATIP2). Decision tree ensembles in the form of a random forest classifier were used to calculate the relative predictive power of these different variables in a multivariate analysis.

Results: The minor IL28B allele (bad risk for treatment response) was significantly associated with increased expression of ISGs, and, unexpectedly, with decreased expression of IL28B. Stratification of the patients into SVR and NR revealed that ISG expression was conditionally independent from the IL28B genotype, i.e. there was an increased expression of ISGs in NR compared to SVR irrespective of the IL28B genotype. The random forest feature score (RFFS) identified IFI27 (RFFS = 2.93), RSAD2 (1.88) and HTATIP2 (1.50) expression and the HCV genotype (1.62) as the strongest predictors of treatment response. ROC curves of the IL28B SNPs showed an AUC of 0.66 with an error rate (ERR) of 0.38 . A classifier with the 3 best classifying genes showed an excellent test performance with an AUC of 0.94 and ERR of 0.15 . The addition of IL28B genotype information did not improve the predictive power of the 3-gene classifier.

Conclusions: IL28B genotype and hepatic ISG expression are conditionally independent predictors of treatment response in CHC. There is no direct link between altered IFN $\lambda 3$ expression and pre-activation of the endogenous system in the liver. Hepatic ISG expression is by far the better predictor for treatment response than IL28B genotype.

Back 